\title{
Impacto de la toma de conciencia en las organizaciones ${ }^{*}$
}

\author{
Impact of awareness on organizations
}

\section{Impacto da conscientização das organizações}

Recibido: 28 de marzo de 2017

Revisado: 22 de mayo de 2017

Aceptado: 8 de junio de 2017

Shirley Katherine Contreras Sanabria***

Escuela Colombiana de Ingeniería Julio Garavito

Lethy Patricia Cruz Ramirez****

Escuela Colombiana de Ingeniería Julio Garavito

Mónica Julieth Cruz Ramírez******

Escuela Colombiana de Ingeniería Julio Garavito

\section{RESUMEN}

El objetivo del presente artículo es determinar los impactos económicos, ambientales y de seguridad y salud en el trabajo, ocasionados por la ausencia de toma de conciencia en las organizaciones. Este artículo es el inicio de una serie de escritos que profundizarán sobre la toma de conciencia, su impacto en las organizaciones, cómo se evalúa, qué acciones preventivas y correctivas debe tomar la organización en busca de la mejora continua, entre otros. La limitación en la realización de este artículo consistió en la escasa bibliografía en el tema propuesto. Las consecuencias de los accidentes de trabajo afectan no solo al trabajador sino también

* $\quad$ Artículo resultado de investigación. DOI: http://dx.doi.org/10.15332/s2145-1389.2017.0001.06

** Especialista en Gestión Integrada QHSE. Ingeniera Industrial. Línea de investigación: gestión de la calidad, medio ambiente, seguridad y salud ocupacional. Escuela Colombiana de Ingeniería Julio Garavito. Correo electrónico: skatherine.contreras@gmail.com.

** Especialista en Gestión Integrada QHSE. Contadora Pública. Línea de investigación: gestión de la calidad, medio ambiente, seguridad y salud ocupacional. Escuela Colombiana de Ingeniería Julio Garavito. Correo electrónico: lpcruzr@davivienda.com, lethycruz@hotmail.com.

**** Especialista en Gestión Integrada QHSE. Fisioterapeuta. Línea de investigación: gestión de la calidad, medio ambiente, seguridad y salud ocupacional. Escuela Colombiana de Ingeniería Julio Garavito. Correo electrónico: monica.cruz@iberoamericana.edu.co, mjcruzr@hotmail.com. 
a sus familias, pueden ocasionar un daño ambiental y una pérdida económica importante para la comunidad, por esta causa es necesario determinar cuáles son los factores que influyen para que estos se presenten. Casos de estudio y experiencias prácticas han demostrado que la frecuencia y la gravedad de los accidentes dependen de los peligros inherentes al trabajo y a factores físicos, fisiológicos y psicológicos propios del trabajador, lo que lleva a concluir que en gran medida las lesiones son producidas por falta de conciencia de los mismos trabajadores (OIT, 2015). Debido al panorama anterior se evidencia la importancia de la toma de conciencia para el desarrollo económico y social del país; desde el punto de vista ambiental, calidad y de seguridad y salud en el trabajo, las acciones de las organizaciones pueden ocasionar riesgos al trabajador, su familia, la sociedad y el medio ambiente, aumentando la susceptibilidad a la ocurrencia de fenómenos naturales peligrosos, pérdidas económicas o situaciones de riesgo que posteriormente se materialicen en desastres. Dada la importancia de la toma de conciencia las normas técnicas NTC-ISO 9001:2008 Sistemas de gestión de la calidad: Requisitos, NTC-ISO 14001:2004 Sistemas de gestión ambiental: Requisitos y NTCOHSAS 18001:2007Sistemas de gestión de la seguridad y la salud ocupacional: Requisitos, la incluyeron como el requisito de: Competencia, formación y toma de conciencia.

Palabras clave: toma de conciencia, calidad, sistemas de gestion, trabajadores.

\section{ABSTRACT}

The aim of this article is to determine the economic, environmental and health and safety at work, caused by the lack of awareness in organizations impacts. This article is the beginning of a series of writings that deepen awareness about its impact on organizations, as assessed, preventive and corrective action should take the organization for continuous improvement, among others. The limitation in conducting this article, consisted of the scant literature on the proposed topic. The consequences of accidents affect not only the workers but their families, can also cause significant environmental damage and economic loss for the community, for this reason it is necessary to identify the factors that influence these arise are. Case studies and practical experience have shown that the frequency and severity of accidents depend on the dangers inherent in work and own physical, physiological and psychological factors worker, leading to the conclusion that largely injuries are caused by lack awareness of these workers (OIT, 2015). Due to the above scenario the importance of awareness for economic and social development of the country is evident; from the environmental point of view, quality and safety and health at work actions organizations can pose risks to the worker, his family, society and the environment; increasing susceptibility to the occurrence of natural hazards, economic loss or risk situations that subsequently materialize disaster. Given the importance of awareness the NTC-ISO 9001:2008 management systems quality, NTC-ISO 14001:2004 environmental management systems and NTC-OHSAS 18001:2007 included in sections 6.2.2, 4.4.2, 4.4.2 competence, training and awareness, respectively.

Keywords: Awareness, quality, management systems, workers.

\section{RESUMO}

O objetivo do presente artigo é determinar os impactos econômicos, ambiental e de segurança e saúde no trabalho, causados pela ausência de conscientização 
nas organizações. Este artigo éo início de um conjunto de artigos escritos que aprofundassem sobre a conscientização, seu impacto nas organizações, o modo de avaliação, ações preventivas e corretivas que a organização deve estabelecer para a melhoria contínua, entre outros. A limitação na realização deste artigo consistiu na escassa literatura no tema proposto. As consequências dos acidentes de trabalho afetam não somente aos trabalhadores, às suas familias, também pode causar danos ambientais e causar importantes prejuízos económicos para a comunidade, por esta razão é necessário identificar quais são os fatores que influenciavam para que estes ocorressem. Casos de estudo e experiências práticas tem demonstrado que a frequência e a gravidade dos acidentes dependem dos perigos inerentes ao trabalho e a fatores físicos, fisiológicos e psicológicos próprios do trabalhador, o que permitirá concluir que em grande medida as lesões são causadas pela falta de consciência dos trabalhadores (OIT, 2015). Devido ao cenário anterior se evidencia a importância da conscientização para o desenvolvimento econômico e social do país; no plano ambiental, de qualidade e segurança e saúde no trabalho, as ações das organizações podem causar riscos para 0 trabalhador, a sua família, a sociedade e 0 meio ambiente; aumentando a suscetibilidade de ocorrências de desastres naturais, perdas econômicas ou situações de risco que posteriormente se materializam em catástrofe. Dada à importância da conscientização as normas NTC 9001:2008 sistemas de gestão da qualidade, NTC ISO 14001: 2004 sistemas de gestão ambiental e NTC OHSAS 18001: 2007 e incluíram-nas seções 6.2.2, 4.4.2, 4.4.2. Habilidade, formação e conscientização, respectivamente.

Palavras-chave: conscientização, qualidade, acidentes de trabalho, trabalhadores, ambiental.

\section{INTRODUCCIÓN}

La toma de conciencia es un proceso gradual que se da cuando el individuo decide modificar, transformar 0 adaptar su paradigma, que se ve reflejado en su forma de actuar, verse a sí mismo y percibir el mundo. La toma de conciencia se obtiene a través de la experiencia, el conocimiento adquirido, comprendiendo cómo se logró y reflexionando sobre el mismo.

Pero, ¿esta toma de conciencia por qué es importante para las organizaciones? La falta de toma de conciencia genera pérdidas de recursos económicos, humanos, ambientales parciales o totales, que afectan el apropiado funcionamiento y crecimiento de las empresas, impactando directamente la economía de los trabajadores, sus familias, las organizaciones y el país.

Implementar la toma de conciencia es un proceso de mejora continua que busca incrementar la eficacia de los sistemas de gestión, reducir el impacto negativo en la salud, la seguridad, el medio ambiente y la calidad. Sin embargo, según la OIT, en el tema "seguridad y salud en el trabajo" publicado en su página web, refiere que cada 15 segundos un trabajador muere a causa de accidentes o enfermedades relacionadas con el trabajo, cada 15 segundos 160 trabajadores tienen un accidente laboral, cada día mueren 6.300 personas a causa de accidentes o enfermedades relacionadas con el trabajo, más de 2.3 millones de muertes por año, casi el doble que las muertes causadas por la guerra y más de las muertes ocasionadas por la malaria. Anualmente ocurren más de 317 millones de accidentes en el trabajo, muchos de estos accidentes resultan en absentismo laboral. El coste de esta adversidad diaria es enorme y la carga económica de las malas prácticas de seguridad y salud se estima en un $4 \%$ del producto interno bruto global de cada año (OIT, 2015), sin estimar aquellas que se producen por los malos procesos en las organizaciones. 
Según el Ministerio de Trabajo, a través de su dirección de riesgos laborales, revela que:

755 personas murieron en Colombia en el 2013 a causa de accidentes de trabajo; en este mismo año murió cada 11 horas y media una persona a causa de un accidente de trabajo; de las 938 muertes reportadas en el 2013 un total de 755 ya fueron calificadas, es decir que plenamente se estableció que perdieron la vida como consecuencia de las tareas laborales que desarrollaban; otras 375 muertes calificadas se registraron en el 2011 y 528 en el 2012. (Mintrabajo, 2014)

El promedio de accidentes de trabajo en Colombia en el año 2013 fue de 62 cada hora, esto es 1.487 diarios, lo que significa 543.079 accidentes de trabajo calificados ocurridos en el país en el 2013, sin embargo, los reportados fueron 656.817, según los procesos calificados, en este año murieron 2 personas por enfermedades laborales, entre tanto, las muertes reportadas fueron 51 (Mintrabajo, 2014).

Desde el punto de vista ambiental, las acciones de las organizaciones pueden ocasionar riesgos a la sociedad, la construcción de infraestructura, la manipulación de materiales o procesos productivos peligrosos, la alteración del medio ambiente, entre muchas otras situaciones, pueden claramente aumentar la susceptibilidad a la ocurrencia de fenómenos naturales peligrosos, consolidando situaciones de riesgo que posteriormente se materialicen en desastres.

Con el fin de minimizar el impacto negativo que pueden ocasionar las organizaciones y personas al medio ambiente, la Constitución en su artículo 95 es explícita en señalar los deberes y responsabilidades de toda persona, con el fin de proteger los recursos culturales y naturales del país y velar por la conservación de un ambiente sano (Asamblea Nacional Constituyente, 1991). Según un anticipo del V Informe Nacional de Biodiversidad, elaborado por el Ministerio de Medio
Ambiente y el Programa de la ONU para el Desarrollo (PNUD), se presentó como sustento los resultados del "Análisis ambiental del país" elaborado por el Banco Mundial (2012), en el que se afirma que la degradación ambiental genera un costo equivalente al 3,5 \% del producto interno bruto (PIB) de Colombia. El documento explica que el daño ambiental en el país se debe a cinco "motores": los cambios en el uso del suelo, la disminución, pérdida o degradación de elementos de los ecosistemas nativos y agro sistemas y las invasiones de especies no nativas. Un cuarto factor es la contaminación y toxificación del agua por la actividad minera, la agricultura industrial y la ganadería y, finalmente, el cambio climático (Revista Dinero, 2014).

El informe explica que el deterioro de los suelos hizo que durante los extremos climáticos de 2010-2011 se destruyera cerca del $14 \%$ de la red vial colombiana y que las inundaciones y deslizamientos afectaran al $7 \%$ de la población (Revista Dinero, 2014).

En este sentido, el informe señala que, a pesar de los inmensos retos de la gestión ambiental, descritos en el Plan Nacional de Desarrollo (2010-2014), "solo se asignó el 0,27 \% del presupuesto total de la nación en el 2009; el 0,28 \% en el 2010 y 2011; el 0,33\% en el 2012 y el 0,39\% en el 2013" (Revista Dinero, 2014).

Estas cifras que afectan la economía, ocasionando pérdidas humanas, económicas y ambientales a las organizaciones públicas, privadas y los gobiernos, se disminuiría con la toma de conciencia en los procesos de calidad ambiental y seguridad y salud en el trabajo.

\section{METODOLOGÍA}

Este artículo se fundamenta en el trabajo de grado "Guía metodológica para evaluar la toma de conciencia 
de los sistemas de gestión ambiental y seguridad y salud en el trabajo", para la especialización Gestión Integrada QHSE de la Escuela Colombia de Ingeniería Julio Garavito; se llevó a cabo un barrido histórico de los conceptos de "conciencia" y "toma de conciencia" desde el punto de vista filosófico, psicológico y neurológico para concluir en la selección de cada definición o en la creación o adaptación de un concepto nuevo de estos dos términos para ser asociados a los sistemas de gestión.

Se realizó la búsqueda de información documental en bases de datos, bibliografía, publicaciones en revistas indexadas, permitiendo el desarrollo de los elementos más importantes asociados a la toma de conciencia, de acuerdo a los requisitos de la norma NTC-ISO 9001:2008, la cual se incluyó y analizó para el presente artículo, y las normas NTC-ISO 14001:2004 y NTCOHSAS 18001:2007.

La investigación fue de tipo exploratorio, debido que se realizó sobre fenómenos de los que se tiene poco o ningún conocimiento, como es el caso de la toma de conciencia, dicha investigación determinará lineamientos para investigaciones futuras.

El presente artículo analiza la conciencia, la toma de conciencia y su impacto en las organizaciones, la guía mencionada anteriormente evalúa y da herramientas para implementar la toma de conciencia y pretendemos darla a conocer en posteriores escritos.

\section{RESULTADOS Y DISCUSIÓN}

La historia de la conciencia en la filosofía se puede distinguir en tres amplias categorías: la primera categoría asocia la conciencia con las actividades y los procesos de conocimiento, la segunda la vincula con la idea de actividad y sensación de sí mismo, mientras que la tercera niega su existencia, función y estudio (Yañez, 2010).

Desde la antigüedad, en la filosofía griega se ha interrogado por la naturaleza de la conciencia, considerada como la característica esencial del ser humano. Distintos filósofos griegos como Platón, Aristóteles y Sócrates intentaron dar respuesta a la pregunta referida a qué es la conciencia y cuál es su naturaleza. Independientemente de sus variaciones conceptuales, lo común en estas primeras formulaciones es la relación estrecha de la conciencia con el concepto de mente, cuya función principal es el conocimiento del mundo (Yañez, 2010).

Dentro de las primeras aproximaciones a la conciencia en los inicios de la psicología encontramos a Wundt $(1911,1912)$, donde la mente era vista como una entidad compuesta de partes que se conectaban de alguna manera gracias a la conciencia. Esta proporcionaba coherencia y organización de los elementos mentales (Yañez, 2010).

Otros conceptos de la escuela europea fueron los de Freud, Piaget, Vygotsky y gran parte de los exponentes de la psicología soviética, donde la conciencia se equiparó a una actividad de conocimiento que en algunos casos podía ejercer algunas funciones de control y de orientación de la acción (Piaget, 1981).

Piaget (1981) señala que la conciencia tenia conexión estrecha con el proceso de conocimiento.

En cuanto a lo indicado por Vygotsky, quien también estudio la conciencia, su propuesta psicológica afrontaba el problema de los procesos cognoscitivos desde una perspectiva histórica y socio-genética (Díaz, 2005). A continuación, se realiza una comparación entre los postulados sobre la conciencia de Piaget y Vygotsky (Díaz, 2005). 
Tabla 1. Comparación entre los postulados sobre la conciencia de Piaget y Vygotsky

\begin{tabular}{|c|c|c|}
\hline Aspecto & PIAGET & VYGOTSKI \\
\hline Definición & $\begin{array}{l}\text { Grado de } \\
\text { conocimiento } \\
\text { y capacidad de } \\
\text { reflexión sobre } \\
\text { mecanismos } \\
\text { cognoscitivos. }\end{array}$ & $\begin{array}{l}\text { Reflejo psíquico de la realidad } \\
\text { y sistema de reglas de } \\
\text { funcionamiento y formación } \\
\text { de conceptos. }\end{array}$ \\
\hline Funciones & $\begin{array}{l}\text { Eliminación } \\
\text { del conflicto } \\
\text { cognoscitivo. }\end{array}$ & $\begin{array}{l}\text { - Mediador representacional. } \\
\text { - Independización de la } \\
\text { realidad externa. } \\
\text { - Regulador de la actividad } \\
\text { cognoscente: planificación, } \\
\text { regulación y control. }\end{array}$ \\
\hline Proceso & $\begin{array}{l}\text { Equilibración } \\
\text { progresiva como } \\
\text { reconstrucción } \\
\text { hacia mayores } \\
\text { niveles de } \\
\text { conciencia. }\end{array}$ & $\begin{array}{l}\text { De la inteligencia práctica a } \\
\text { la formación de conceptos. } \\
\text { Interviene en la objetivización } \\
\text { a partir de la actividad } \\
\text { transformando la actividad } \\
\text { adaptativa en representativa. }\end{array}$ \\
\hline Naturaleza & $\begin{array}{l}\text { Conceptual y } \\
\text { representacional. }\end{array}$ & $\begin{array}{l}\text { Representacional (signos) } \\
\text { histórica y socio genética }\end{array}$ \\
\hline Aparición & $\begin{array}{l}\text { Etapas superiores } \\
\text { del desarrollo } \\
\text { cognoscitivo. }\end{array}$ & $\begin{array}{l}\text { Etapas superiores del } \\
\text { desarrollo cognoscitivo }\end{array}$ \\
\hline
\end{tabular}

Fuente: Conciencia y metacognición (Díaz, 2005).

Desde el punto de vista neurológico, el ser humano es cuerpo consciente, su conciencia lo lleva a estar en constante relación con el mundo. En esta relación la subjetividad y la objetividad forman una relación dialéctica en la que se genera un conocer solidario con el actuar y viceversa (Molina, 2004). Otras teorías que se destacan acerca de la conciencia en el ámbito neurológico son:

- Según Dainton (2008) citado por Yañez (2010) la conciencia consiste en una sucesión de fragmentos o pulsos de experiencia, de punta a punta como si fuesen una fila de ladrillos, a menos que olvide el cemento que los une.

- Llinas (2003) citado por Yañez (2010) propone la conciencia como correlatos de la actividad neuronal, las oscilaciones neuronales de 40 hercios dados en los centros corticales y subcorticales serían los responsables de la conciencia, ya que en los momentos en que un sujeto discrimina un estímulo se presentan estas zonas activadas de manera cíclica.

- Según la teoría de Hameroff y Penrose (1994) citado por Bejar (2007), quienes publicaron en su obra Sombras de la mente la cual está dedicada especialmente a la conciencia, proponen la teoría de ORSH OR (Reducción Objetiva Orquestada), sugiriendo que las ondas cerebrales se derivan de vibraciones de microtúbulos en un profundo nivel, y que desde un punto de vista práctico, el tratamiento de las vibraciones de los microtúbulos del cerebro podría beneficiar el tratamiento de una gran cantidad de trastornos mentales, neurológicos y cognitivos (Elsevier, 16 de enero de 2014).

La preocupación por la toma de conciencia se expresa en diferentes ámbitos académicos e investigativos con diferentes términos. De una manera muy general y por lo demás atrevida, se insiste permanentemente en estudiar lo que piensan, estructuran o traen en su mente los sujetos y colocan a disposición en el momento de aprender, estudiar, compartir, etcétera. Particularmente en lo relacionado con la toma de conciencia, es un instrumento para construir renovado pensamiento (Taborda, 2010).

Lo anterior evidencia cómo la conciencia y su significado han sido cuestionados desde siempre, y cómo su concepción ha cambiado a través del tiempo y aún continúa siendo motivo de investigación y debate en los diferentes aspectos de la humanidad.

Esta exhaustiva investigación permitió definir la conciencia como el conocimiento de sí mismo, ancestral y del mundo, que se obtiene mediante procesos inconscientes, experiencia e información compartida de diferentes áreas del cerebro. La conciencia no es estática, 
cambia y se transforma en el tiempo de manera gradual a través de los comportamientos, la adquisición de experiencias y la reflexión de las mismas.

Y la toma de conciencia como un proceso gradual que se da cuando el individuo decide modificar, transformar 0 adaptar su paradigma, que se ve reflejado en su forma de actuar, verse a sí mismo y percibir el mundo. La toma de conciencia se obtiene a través de la experiencia, el conocimiento adquirido, comprendiendo cómo se logró y reflexionando sobre el mismo.

Estas dos definiciones tienen sinergia, determinados en que la conciencia y la toma de conciencia son cambiantes, adaptables y transformables mediante la experiencia adquirida. La conciencia es el conocimiento de sí mismo y para que exista toma de conciencia se requiere tener la disposición de cambiar, modificar 0 transformar las acciones o pensamientos y reflexionar cómo se llegó al logro.

Por ejemplo, un atleta profesional que requiera mejorar su desempeño debe tomar conciencia de sus movimientos al correr y optimizarlos logrando mejorar su rendimiento deportivo. Una vez adquirida la experiencia necesaria, estos movimientos son conscientes y a través del tiempo se convierten en rutinarios e inconscientes.

De otra parte, la alta relevancia de la toma de conciencia se expresa en los sistemas integrados de gestión, sin embargo, las normas ISO no profundizan en cómo mejorar, adquirir o evaluar la toma de conciencia y al querer implementarlo se encuentra tanta literatura y tan diversa (puntos de vista psicológico, neurológico, filosófico, entre otros) que las organizaciones pueden perder el norte y seguir implementando procesos insuficientes e ineficaces.

Dada la importancia de la toma de conciencia, la norma ISO 9000:2008 estableció un requisito en su numeral
6.2.2, competencia, formación y toma de conciencia, donde expresa que "la organización debe asegurarse de que su personal es consciente de la pertinencia e importancia de sus actividades".

La norma ISO 14001:2004 estableció un requisito en su numeral 4.4.2, competencia, formación y toma de conciencia, donde expresa que "la organización debe establecer uno o varios procedimientos para que sus empleados o las personas que trabajan en su nombre tomen conciencia de...", y en el anexo A (informativo) A.4.2 refiriéndose a "la toma de conciencia, el conocimiento, la comprensión y la competencia se pueden obtener 0 mejorar a través de formación, educación 0 experiencia laboral".

En cuanto a la norma OHSAS 18001:2007, se estableció en el requisito 4.4.2, competencia, formación y toma de conciencia, refiriéndose a ella sobre como "la organización debe establecer, implementar y mantener un(os) procedimiento(s) para establecer que las personas que trabajan bajo su control tomen conciencia de...".

En los anteriores numerales y anexo de cada una de las normas, la toma de conciencia se expresa de manera explícita. No obstante lo anterior, al realizar el análisis de la totalidad de los requisitos de las normas ISO 9001:2008, ISO 14001:2004 y OHSAS 18001:2007, se identifica que la toma de conciencia aplicaría para algunos requisitos de manera directa, porque están asociados a los conceptos definidos de "conciencia" y "toma de conciencia" indicados anteriormente, aunque en estas obligaciones no se incluya o exprese de manera directa la "toma de conciencia".

A continuación se analizó la aplicación o no (N/A) de la toma de conciencia en cada uno de los numerales de las normas ISO 9001:2008, ISO 14001:2004 y OHSAS 18001:2007, con el fin de orientar a las organizaciones para la implementación de la misma. 
Shirley Katherine Contreras Sanabria, Lethy Patricia Cruz Ramírez, Mónica Julieth Cruz Ramírez

Tabla 2. Correlación de los criterios de las normas ISO 9001:2008, ISO 14001:2004 y OSHAS 18001:2007 con respecto a la toma de conciencia

\begin{tabular}{|c|c|c|c|c|c|c|c|c|}
\hline \multicolumn{2}{|r|}{ ISO 9001:2008 } & \multirow{2}{*}{$\begin{array}{c}\text { Toma de } \\
\text { conciencia }\end{array}$} & \multicolumn{2}{|c|}{ ISO 14001:2004 } & \multirow{2}{*}{$\begin{array}{c}\text { Toma de } \\
\text { conciencia }\end{array}$} & \multicolumn{2}{|c|}{ OHSAS 18001:2007 } & \multirow{2}{*}{$\begin{array}{c}\text { Toma de } \\
\text { conciencia } \\
\text { N/A }\end{array}$} \\
\hline 4.1 & Requisitos generales & & 4.1 & $\begin{array}{l}\text { Requisitos } \\
\text { generales }\end{array}$ & & 4.1 & $\begin{array}{l}\text { Requisitos } \\
\text { generales }\end{array}$ & \\
\hline $\begin{array}{c}5.1 \\
5.3 \\
8.5 .1\end{array}$ & $\begin{array}{l}\text { Compromiso de la } \\
\text { dirección } \\
\text { Política de la calidad } \\
\text { Mejora continua }\end{array}$ & $\mathrm{N} / \mathrm{A}$ & 4.2 & Política ambiental & $\mathrm{N} / \mathrm{A}$ & 4.2 & Política de S Y SO & $\mathrm{N} / \mathrm{A}$ \\
\hline 5.4 & \multicolumn{2}{|l|}{ Planificación (solo título) } & 4.3 & \multicolumn{2}{|c|}{ Planificación (solo título) } & 4.3 & \multicolumn{2}{|c|}{ Planificación (solo título) } \\
\hline $\begin{array}{c}5.2 \\
7.2 .1 \\
\\
7.2 .2\end{array}$ & $\begin{array}{l}\text { Enfoque al cliente } \\
\text { Determinación de los } \\
\text { requisitos relacionados } \\
\text { con el producto } \\
\text { Revisión de los } \\
\text { requisitos relacionados } \\
\text { con el producto }\end{array}$ & $\mathrm{N} / \mathrm{A}$ & 4.3 .1 & $\begin{array}{l}\text { Aspectos } \\
\text { ambientales }\end{array}$ & $\mathrm{N} / \mathrm{A}$ & 4.3.1. & $\begin{array}{l}\text { Identificación de } \\
\text { peligros, valoración } \\
\text { de riesgos y } \\
\text { determinación de } \\
\text { los controles }\end{array}$ & N/A \\
\hline $\begin{array}{c}5.2 \\
7.2 .1\end{array}$ & $\begin{array}{l}\text { Objetivos de la calidad } \\
\text { Determinación de los } \\
\text { requisitos relacionados } \\
\text { con el producto }\end{array}$ & $\mathrm{N} / \mathrm{A}$ & 4.3 .2 & $\begin{array}{l}\text { Requisitos legales y } \\
\text { otros requisitos }\end{array}$ & N/A & 4.3 .2 & $\begin{array}{l}\text { Requisitos legales y } \\
\text { otros requisitos }\end{array}$ & $\mathrm{N} / \mathrm{A}$ \\
\hline $\begin{array}{l}5.4 .1 \\
5.4 .2 \\
8.5 .1\end{array}$ & $\begin{array}{l}\text { Objetivos de la calidad } \\
\text { Planificación del } \\
\text { sistema de gestión de } \\
\text { la calidad } \\
\text { Mejora Continua }\end{array}$ & SI & 4.3.3. & $\begin{array}{l}\text { Objetivos, metas y } \\
\text { programas }\end{array}$ & SI & 4.3.3. & $\begin{array}{l}\text { Objetivos y } \\
\text { programa(s) }\end{array}$ & SI \\
\hline 7 & Realización del product & (solo título) & 4.4 & $\begin{array}{l}\text { Implementación y of } \\
\text { título) }\end{array}$ & ción (solo & 4.4 & $\begin{array}{l}\text { Implementación y o } \\
\text { título) }\end{array}$ & ración (solo \\
\hline $\begin{array}{r}5.1 \\
5.5 .1 \\
5.5 .2 \\
6.1 \\
6.3\end{array}$ & $\begin{array}{l}\text { Compromiso de la } \\
\text { dirección } \\
\text { Responsabilidad y } \\
\text { autoridad } \\
\text { Representante de la } \\
\text { dirección } \\
\text { Provisión de recursos } \\
\text { Infraestructura }\end{array}$ & SI & 4.4.1 & $\begin{array}{l}\text { Recursos, } \\
\text { funciones, } \\
\text { responsabilidad y } \\
\text { autoridad }\end{array}$ & SI & 4.4 .1 & $\begin{array}{l}\text { Recursos, } \\
\text { funciones, } \\
\text { responsabilidad, } \\
\text { rendición de } \\
\text { cuentas y autoridad }\end{array}$ & SI \\
\hline $\begin{array}{l}6.2 .1 \\
6.2 .2\end{array}$ & $\begin{array}{l}\text { (Recursos humanos) } \\
\text { Generalidades } \\
\text { Competencia, } \\
\text { formación y toma de } \\
\text { conciencia }\end{array}$ & SI & 4.4 .2 & $\begin{array}{l}\text { Competencia, } \\
\text { formación y toma } \\
\text { de conciencia }\end{array}$ & SI & 4.4 .2 & $\begin{array}{l}\text { Competencia, } \\
\text { formación y toma } \\
\text { de conciencia }\end{array}$ & SI \\
\hline
\end{tabular}


Impacto de la toma de conciencia en las organizaciones

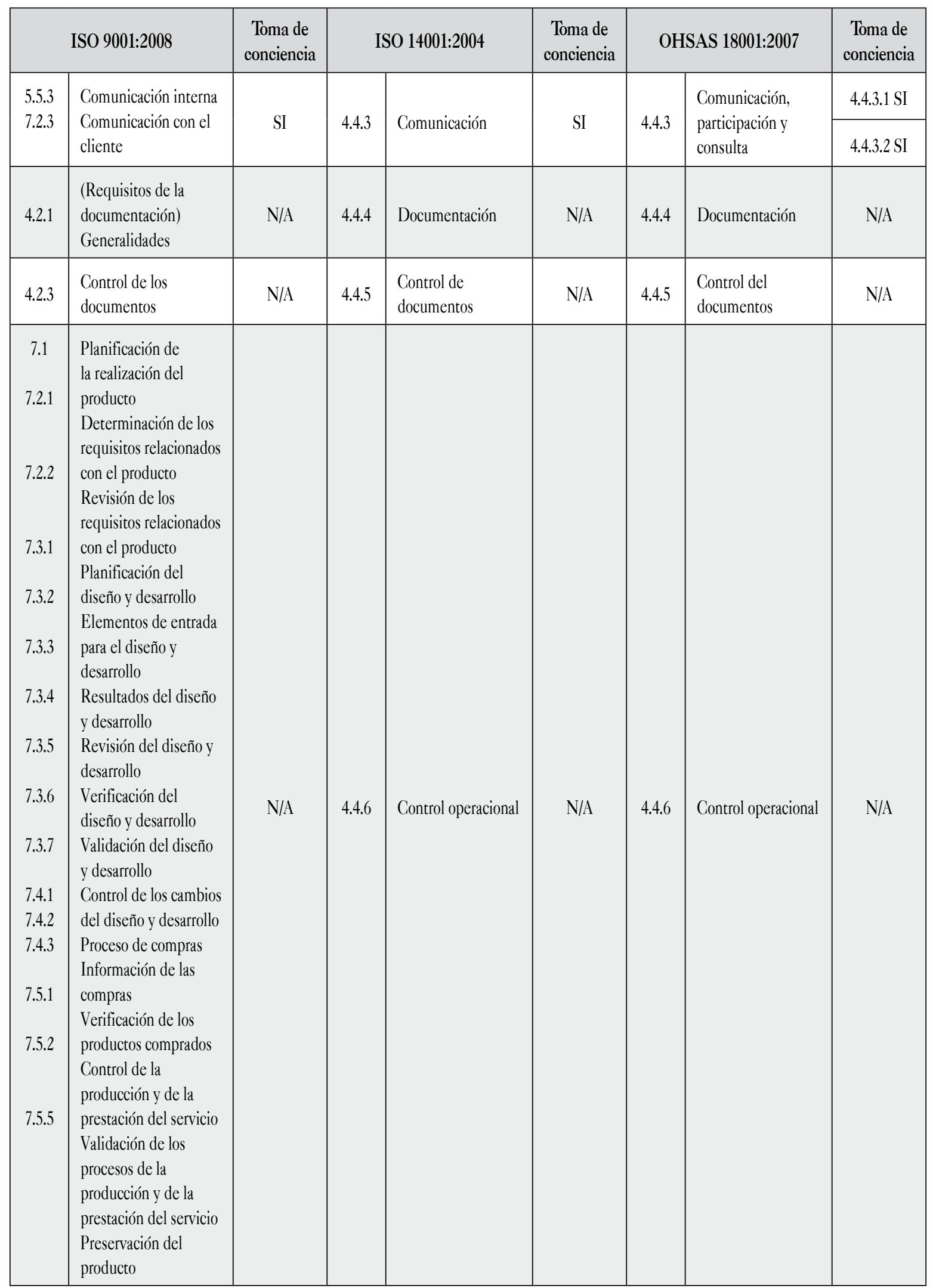


Shirley Katherine Contreras Sanabria, Lethy Patricia Cruz Ramírez, Mónica Julieth Cruz Ramírez

\begin{tabular}{|c|c|c|c|c|c|c|c|c|}
\hline \multicolumn{2}{|r|}{ ISO 9001:2008 } & \multirow{2}{*}{$\begin{array}{c}\text { Toma de } \\
\text { conciencia }\end{array}$} & \multicolumn{2}{|c|}{ ISO 14001:2004 } & \multirow{2}{*}{$\begin{array}{c}\text { Toma de } \\
\text { conciencia }\end{array}$} & \multicolumn{2}{|c|}{ OHSAS 18001:2007 } & \multirow{2}{*}{$\begin{array}{c}\begin{array}{c}\text { Toma de } \\
\text { conciencia }\end{array} \\
\text { SI }\end{array}$} \\
\hline 8.3 & $\begin{array}{l}\text { Control del producto } \\
\text { no conforme }\end{array}$ & & 4.4.7 & $\begin{array}{l}\text { Preparación y } \\
\text { respuesta ante } \\
\text { emergencias }\end{array}$ & & 4.4.7 & $\begin{array}{l}\text { Preparación y } \\
\text { respuesta ante } \\
\text { emergencias }\end{array}$ & \\
\hline 8 & \multicolumn{2}{|c|}{$\begin{array}{l}\text { Medición, análisis y mejora } \\
\text { (título solamente) }\end{array}$} & 4.5 & \multicolumn{2}{|c|}{ Verificación (solo título) } & 4.5 & \multicolumn{2}{|l|}{ Verificación (solo título) } \\
\hline $\begin{array}{c}7.6 \\
8.1 \\
8.2 .3 \\
8.2 .4 \\
8.4\end{array}$ & $\begin{array}{l}\text { Control de los equipos } \\
\text { de seguimiento y } \\
\text { medición } \\
\text { (Medición, análisis y } \\
\text { mejora) generalidades } \\
\text { Seguimiento y } \\
\text { medición de los } \\
\text { procesos } \\
\text { Seguimiento y } \\
\text { medición del producto } \\
\text { Análisis de datos }\end{array}$ & $\mathrm{N} / \mathrm{A}$ & 4.5.1 & $\begin{array}{l}\text { Seguimiento y } \\
\text { medición }\end{array}$ & N/A & 4.5.1 & $\begin{array}{l}\text { Medición y } \\
\text { seguimiento del } \\
\text { desempeño }\end{array}$ & N/A \\
\hline $\begin{array}{l}8.2 .3 \\
8.2 .4\end{array}$ & $\begin{array}{l}\text { Seguimiento y } \\
\text { medición de los } \\
\text { procesos } \\
\text { Seguimiento y } \\
\text { medición del producto }\end{array}$ & $\mathrm{N} / \mathrm{A}$ & 4.5 .2 & $\begin{array}{l}\text { Evaluación del } \\
\text { cumplimiento legal }\end{array}$ & $\mathrm{N} / \mathrm{A}$ & 4.5 .2 & $\begin{array}{l}\text { Evaluación del } \\
\text { cumplimiento legal }\end{array}$ & $\mathrm{N} / \mathrm{A}$ \\
\hline 8.3 & $\begin{array}{l}\text { Control del producto no } \\
\text { conforme }\end{array}$ & & & & & 4.5.3 & $\begin{array}{l}\text { Investigación de } \\
\text { incidentes, no } \\
\text { conformidades, } \\
\text { acciones correctivas y } \\
\text { preventivas }\end{array}$ & SI \\
\hline $\begin{array}{c}8.4 \\
8.5 .2 \\
8.5 .3\end{array}$ & $\begin{array}{l}\text { Analisis de datos } \\
\text { Acción correctiva } \\
\text { Acción preventiva }\end{array}$ & SI & & & & 4.5.3.1 & $\begin{array}{l}\text { Investigación de } \\
\text { incidentes }\end{array}$ & SI \\
\hline & & & 4.5 .3 & $\begin{array}{l}\text { No conformidad, } \\
\text { acción correctiva y } \\
\text { acción preventiva }\end{array}$ & SI & 4.5.3.2 & $\begin{array}{l}\text { No conformidad, } \\
\text { acción correctiva y } \\
\text { acción preventiva }\end{array}$ & SI \\
\hline 4.2 .4 & Control de los registros & N/A & 4.5.4 & $\begin{array}{l}\text { Control de los } \\
\text { registros }\end{array}$ & N/A & 4.5.4 & $\begin{array}{l}\text { Control de los } \\
\text { registros }\end{array}$ & $\mathrm{N} / \mathrm{A}$ \\
\hline 4.5 .5 & Auditoría interna & SI & 4.5 .5 & Auditoría interna & SI & 4.5 .5 & Auditoría interna & SI \\
\hline $\begin{array}{c}5.1 \\
5.6 \\
5.6 .1 \\
5.6 .2 \\
\\
5.6 .3 \\
8.5 .1\end{array}$ & $\begin{array}{l}\text { Compromiso de la } \\
\text { dirección } \\
\text { Revisión por la dirección } \\
\text { (título solamente) } \\
\text { Generalidades } \\
\text { Información de entrada } \\
\text { para la revisión } \\
\text { Resultados de la } \\
\text { revisión } \\
\text { Mejora continua }\end{array}$ & N/A & 4.6 & $\begin{array}{l}\text { Revisión por la } \\
\text { dirección }\end{array}$ & $\mathrm{N} / \mathrm{A}$ & 4.6 & $\begin{array}{l}\text { Revisión por la } \\
\text { dirección }\end{array}$ & N/A \\
\hline
\end{tabular}

Fuente: elaboración propia 
Con respecto a la tabla 2 "Correlación de los criterios de las normas ISO 9001:2008, ISO 14001:2004 y OSHAS 18001:2007 con respecto a la toma de conciencia”, se analizó que:

Todos los requisitos de las normas NTC-ISO 9001:2008, NTC-ISO 14001:2004 y NTC-OHSAS 18001:2007 que tengan inmersos en ellos participación y comunicación hacen parte del proceso de toma de conciencia, debido a que cuando un trabajador participa en diversas actividades y adquiere conocimiento a través de la comunicación, logra experiencia que posteriormente le permite reflexionar sobre la misma, creando en él un cambio gradual en su forma de actuar.

Los sistemas de gestión integrados al ser procesos graduales dentro de las organizaciones, van de la mano del proceso de toma de conciencia, porque requieren la adquisición de nuevos conocimientos, reflexión que genera cambio en el actuar y con el cual se incrementa la experiencia, permitiendo así la mejora continua.

El éxito del cumplimiento de la política y los objetivos de la organización depende de la toma de conciencia de sus trabajadores, debido a que quienes hacen que estos se cumplan son ellos mismos, mediante su actuación, aplicando el conocimiento, experiencia, habilidades y aptitudes para el desarrollo de su labor.

Los diferentes planes y programas de las normas requieren participación activa de los trabajadores en los diferentes niveles, es decir, cuando el trabajador participa se vuelve más consciente de las acciones a tomar: mejorando procesos, evitando, controlando y minimizando incidentes de $\mathrm{S}$ y $\mathrm{SO}$ e impactos ambientales negativos según sea el caso; por ejemplo, en los programas de preparación y respuesta ante emergencias se requiere adquirir conocimiento sobre los procedimientos, modificar o transformar las conductas y la realización de pruebas periódicas que son generadoras de experiencia, permitiendo reflexionar sobre la forma de actuar y el resultado que se obtiene.

Otro ejemplo es el criterio de participación y consulta, donde los trabajadores por el conocimiento adquirido en sus labores diarias o rutinarias, son quienes realizan la identificación del peligro y participan en la valoración de riesgos y en la determinación de controles de $\mathrm{S}$ y SO, adquiriendo experiencia y transformando o modificando su entorno y su forma de hacer las cosas.

Con respecto al control operacional, este no hace parte de la toma de conciencia, debido a que son procesos estandarizados aplicados por los funcionarios, pero no generan reflexión de los mismos y cuando hay desviaciones se producen incidentes.

Este análisis corrobora que la toma de conciencia se encuentra inmersa en varios de los numerales de las normas ISO mencionadas, y su aplicación ayudará a alcanzar la misión y la visión de las organizaciones.

\section{CONCLUSIONES}

Los sistemas de gestión integrados de las normas NTC-ISO 9001:2008, NTC-ISO 14001:2004 y NTCOHSAS 18001:2007 al ser procesos graduales dentro de las organizaciones, van de la mano del proceso de toma de conciencia, dado que requieren la adquisición de nuevos conocimientos, reflexión que genera cambio en el actuar y con el cual se incrementa la experiencia, permitiendo asíla mejora continua.

El éxito del cumplimiento de la política y los objetivos de la organización depende de la toma de 
conciencia de sus trabajadores, debido a que quienes hacen que estos se cumplan son ellos mismos, mediante su actuación, aplicando el conocimiento, experiencia, habilidades y aptitudes para el desarrollo de su labor.

Para promover la toma de conciencia y a través de ella generar cambios en los paradigmas y en la forma de actuar de los empleados. Se debe tener en cuenta que:

- El individuo debe tomar la decisión de modificar sus hábitos.

- La toma de conciencia se logra mediante un proceso gradual del individuo y la organización.

- Los planes de mejora deben incluir actividades donde:

- Se adquiera conocimiento.

- Haya experiencias vivenciales tales como pruebas, planes de evacuación, simulacros, entre otros.

- Se genere reflexión sobre eventos experimentados por otros miembros de la organización con respecto a situaciones que hayan afectado los sistemas de gestión de calidad, ambiental y seguridad y salud en el trabajo.

Las autoras del presente artículo recomiendan que la toma de conciencia sea aplicada a todos los tipos de organización (servicios, manufactura, públicos, privados, entre otros), evaluando así la experiencia, el conocimiento adquirido, la reflexión y el cambio en la forma de actuar de los trabajadores, de verse a sí mismo y de percibir el mundo y de esta manera determinar la madurez de los sistemas de gestión.

\section{REFERENCIAS}

Banco Internacional de Reconstrucción y Fomento / Banco Mundial. (Marzo de 2012). Unidad Nacional para la gestión del riesgo de desastres en Colombia: un aporte para la construcción de políticas públicas. Recuperado de http://gestiondelriesgo.gov.co/ sigpad/archivos/GESTIONDELRIESGOWEB. pdf página 8.

Asamblea Nacional Constituyente. (4 de julio de 1991). Constitución Política de Colombia. Bogotá, Colombia.

Banco Internacional de Reconstrucción y Fomento / Banco Mundial. (Marzo de 2012). Unidad Nacional para la gestión del riesgo de desastres - Colombia. Obtenido de Análisis de la gestión del riesgo de desastres en Colombia:: http://gestiondelriesgo.gov.co/sigpad/archivos/GESTIONDELRIESGOWEB.pdf

Bejar, M. (3 de febrero de 2007). Penrose sienta las bases de una biofísica cuántica de la mente. $R \boldsymbol{\ell}$ vista Electrónica de Ciencia, Tecnología, Sociedad y Cultura. Recuperado de http://www.tendencias21. net/Penrose-sienta-las-bases-de-una-biofisicacuantica-de-la-mente_a1406.html

Icontec. (24 de noviembre de 2007). Norma Técnica Colombiana NTC-OHSAS 18001. Sistemas de Gestión en Seguridady Salud Ocupacional. Requisitos. Bogotá, Colombia: Icontec.

Icontec. (14 de noviembre de 2008). Norma Técnica Colombiana NTC-ISO 9001. Sistemas de Gestión de la Calidad. Requisitos. Bogotá, Colombia: Icontec.

Icontec. (1 de diciembre de 2004). Norma Técnica Colombiana NTC-ISO 14001. Sistemas de Gestión 
Ambiental Requisitos con orientación para su uso. Bogotá, Colombia: Icontec.

Ley 1562 de 2012. (7 de noviembre). Por la cual se modifica el Sistema de Riesgos Laborales y se dictan otras disposiciones en materia de Salud Ocupacional. Diario Oficial n. ${ }^{\circ} 48488$. Recuperado de http://www.alcaldiabogota.gov.co/sisjur/normas/ Norma1.jsp?i $=48365$

Resolución 1401. (14 de mayo de 2007). Por la cual se reglamenta la investigación de incidentes y accidentes de trabajo. Diario Oficial $n^{\circ}$ 46.638. Recuperado de http://www.alcaldiabogota.gov.co/ sisjur/normas/Norma1.jsp?i=53497

Decreto número 1443 (31 de julio de 2014). Por el cual se dictan disposiciones para la implementación del sistema de gestión de seguridad y salud en el trabajo (SG-SST). Diario Oficial $n^{\circ} 49.229$. Recuperado de http://www.alcaldiabogota.gov. co/sisjur/normas/Norma1.jsp?i=58841 Bogotá, Colombia.

Elsevier. (16 de enero de 2014). Discovery of quantum vibrations in 'microtubules' inside brain neurons supports controversial theory of consciousness. ScienceDaily. Recuperado de http://www.sciencedaily. com/releases/2014/01/140116085105.htm
Mintrabajo. (28 de febrero de 2014). La protección en riesgos laborales mas que una obligación una necesidad. Obtenido de http://www.mintrabajo.gov. co/febrero-2014/3065-la-proteccion-en-riesgoslaborales-mas-que-una-obligacion-una-necesidad. html

Molina, M. L. (2004). Modelos de intervención asistencial, socioeducativo y terapéutico en trabajo social. San José: La Universidad de Costa Rica.

OIT. (15 de enero de 2015). Seguridad y salud en el trabajo. Recuperado de http://www.ilo.org/global/topics/ safety-and-health-at-work/lang--es/index.htm

Piaget, J. (1981). La toma de conciencia. Madrid, España: Ediciones Morata S. A.

Revista Dinero. (5 de diciembre de 2014). Hay deterioro ambiental en Colombia. Recuperado de http://www.dinero.com/pais/articulo/dano -medio-ambiente-colombia/196017

Taborda, J. (2010). Relación de la formación y el mecanismo Piagetiano de toma de conciencia. Revista Latinoamericana de estudios educativos, 6(2), 109-128.

Yañez, J. (2010). Cognición y conciencia. Bogotá: Minuto de Dios. 